provided fully accurate and comprehensive information on chlamydia.

Conclusion The marked variation in content, quality and accuracy of available smartphone apps combined with the relatively high proportion that contain harmful information, significantly risks undermining the potential health benefits of an e-health approach to sexual health promotion and information. Disclosure of interest statement Nothing to declare.

\section{P12.04 THE EVIDENCE FOR SEXUALLY TRANSMITTED INFECTIONS AS A MARKER FOR CHILD SEXUAL ABUSE: THE PHYSICAL SIGNS OF CHILD SEXUAL ABUSE $2^{\text {ND }}$ EDITION 2015}

Karen Rogstad*, Amanda Thomas, Neil McIntosh, Cindy Christian, on behalf of The Project Board, STI Working Group of The Royal College of Paediatrics, Child Health, The Royal College of Physicians of London, it's Faculty of Forensic, Legal Medicine. The Physical signs of child sexual abuse an updated evidence-based review, guidance for best practice (2015). Sheffield Teaching Hospitals Foundation Trust and University of Sheffield Medical School, Leeds Community Healthcare NHS Trust, University of Edinburgh, The Children's Hospital of Philadelphia (USA), The Perelman School of Medicine, University of Pennsylvania (USA)

\subsection{6/sextrans-2015-052270.484}

Introduction In 2008 the RCPCH in collaboration with RCP (London) and Faculty of Forensic and Legal Medicine produced the only evidence based review on Physical Signs of Child Sexual Abuse (CSA). The evidence has been used in child protection cases including family and criminal proceedings in the UK and advised practitioners internationally. A further review of evidence with additional representation from American Academy of Paediatrics was undertaken to determine if changes to evidence statements are required.

Methods The 2008 search strategy identified all published primary research literature reporting STIs in relation to child sexual abuse or in children selected for non-abuse. For the 2015 publication, the search strategies were re-run on MEDLINE and EMBASE databases (Jan 2007-March 2014). Studies were selected according to certainty and quality of STI and CSA diagnosis.

Results Two additional studies were identified. One for HPV strengthened the evidence base for external genital warts (EGW) as a marker of CSA, reporting $50 \%$ of children sexually abused. The evidence base is "a significant proportion (31-58\%) have been abused and a revised recommendation to refer children $<13$ yrs for child protection assessment.

One study was for Neisseria gonorrheae also supported the previous evidence statement (sexual abuse reported in 36-83\%). The evidence has not changed significantly; GC, CT, and TV are most likely sexually transmitted and for children with HPV a significant number are sexually transmitted. Referral to child protection services is advised for all under $13 \mathrm{yr}$ olds with GC, CT, TV, EGW; and for syphilis, HIV/Hepatitis $\mathrm{B} / \mathrm{C} /$ Herpes genitalis sexual abuse should always be considered if other modalities have been excluded; infection in the mother does not exclude CSA.

Conclusion Children under 13 yrs presenting with an STIs should have CSA considered and be referred for a child protection assessment unless (rarely) evidence to the contrary.

Conflict of interest KER has received sponsorship, speakers and consultancy fees from Pharma related to HIV therapy and HPV vaccines.

\section{P12.05 OUTREACH CHLAMYDIA TESTING: UPSKILLING A MULTIDISCIPLINARY WORKFORCE}

${ }^{1,2}$ DM Tilley*, ${ }^{3}$ BR Dailey, ${ }^{1} \mathrm{NC}$ Sharp, ${ }^{3} \mathrm{D}$ House. ${ }^{1}$ RPA Sexual Health, Community Health, Sydney Local Health District, Sydney, Australia; ${ }^{2}$ Women's Health Service, Community Health, Sydney Local Health District, Sydney, Australia; ${ }^{3} H I V$ and Related Programs Health Promotion, Community Health, Sydney Local Health District, Sydney, Australia

\subsection{6/sextrans-2015-052270.485}

Introduction Chlamydia is the most common notifiable sexually transmitted infection (STI) in Australia, mostly affecting people aged 29 years and under. Offering testing in an outreach setting is an effective strategy for engaging young people and reducing the number of undiagnosed infections. Our service developed a model for Aboriginal Health Education Officers (HEOs) and Health Promotion Officers (HPOs) to offer urine chlamydia and gonorrhoea testing at community events. To enhance knowledge, confidence and skills, the Clinical Nurse Consultant and other specialist clinicians developed a comprehensive training package. The package includes an operations manual, lesson plan, presentation, role play scenarios, checklists, knowledge quiz and competency assessment. Topics include confidentiality, assessing risk and specimen collection procedures. Ongoing support from the clinical service is provided.

Methods Participants completed a pre and post training survey to measure knowledge and confidence in undertaking urine chlamydia and gonorrhoea testing in an outreach setting. The survey asked for responses to six statements on a scale of 1 (not at all) to 5 (completely). The average scores for each statement pre and post were calculated.

Results A total of nine staff completed the training between August 2014 and January 2015. The staff were from varying disciplines including Aboriginal HEO, HPOs, social work and student nursing. Eight pre and post surveys were completed. For all statements there was an increase in the average score in the post survey compared with the pre survey. The greatest differences were in the statements relating to assessing risk of harm in a young person and use of standard precautions.

Conclusion Participation in the comprehensive training demonstrated an increase in knowledge, confidence and skills of nonclinical health workers to undertake urine chlamydia and gonorrhoea testing in an outreach setting. The training is appropriate for staff from a variety of disciplines including Aboriginal HEOs, HPOs and social workers.

Disclosure of interest statement No disclosures of interest.

\section{P12.06 GENITAL TRICHOMONAS VAGINALIS IS RARE AMONG FEMALE ATTENDEES AT A SYDNEY METROPOLITAN SEXUAL HEALTH CLINIC}

${ }^{1,2}$ DM Tilley*, ${ }^{3} \mathrm{SM}$ Dubedat, ${ }^{4} \mathrm{P}$ Lowe, ${ }^{1,5,6} \mathrm{DJ}$ Templeton. ${ }^{1}$ RPA Sexual Health, Community Health, Sydney Local Health District, Sydney, Australia; 'Women's Health Service, Community Health, Sydney Local Health District, Sydney, Australia; ${ }^{3}$ Department of Microbiology, Royal Prince Alfred Hospital, Sydney, Australia; ${ }^{4}$ Hologic (Australia) Pty Ltd; ${ }^{5}$ The Kirby Institute, University of New South Wales, Sydney, Australia; ${ }^{6}$ Central Clinical School, The University of Sydney, Sydney, Australia

\subsection{6/sextrans-2015-052270.486}

Introduction Trichomonas vaginalis (TV) is the most common non-viral sexually transmitted infection worldwide. Among Australian women, a wide variation in prevalence $(0.38 \%-8.4 \%)$ using nucleic acid amplification tests (NAAT) has been reported. 